\title{
Éloge de la pause : aperçu des signes de ponctuation dans les Festes nouvelles manuscrites et imprimées (XIV ${ }^{\mathrm{e}}-\mathrm{XVI}^{\mathrm{e}}$ siècles)
}

\author{
Yunhao NA \\ Peking University \\ yunhaona@gmail.com \\ https://orcid.org/0000-0001-8281-6035
}

\section{Resumen}

Como fenómeno histórico, la puntuación forma parte, a priori, de los estudios diacrónicos. El examen de la variación en las pausas marcadas en un mismo texto a través de sus diferentes copias contemporáneas y sucesivas puede mostrar tanto la diversidad pragmática como la evolución diacrónica de la señalización de segmentos textuales. En este artículo, se estudia a través de un corpus de textos narrativos, el empleo concreto de los signos de puntuación y su sistematización evolutiva. Buscamos así contribuir a una teoría de la puntuación extensa, que contribuya al estudio de la sintaxis, la ortografía, la lexicología diacrónica o la genética textual.

Palabras clave: puntuación medieval, segmentación, variante, diacronía, hagiografía.

\section{Résumé}

En tant que phénomène historique, la ponctuation fait a priori l'objet d'études diachroniques. L'examen de la variation des pauses marquées dans les témoins contemporains et successifs d'un même texte peut faire ressortir la diversité pragmatique comme l'évolution chronologique du marquage des segments de textes. S'intéressant à une série de textes narratifs, la présente étude a l'objectif non seulement d'analyser des emplois concrets de signes et de leur systématisation évolutive, mais aussi de contribuer à l'élaboration théorique pour orienter l'étude de la ponctuation vers une utilité plus étendue, pouvant servir, à la syntaxe et à l'histoire de l'orthographe comme à la lexicologie diachronique et à la genèse des textes.

Mots-clés : ponctuation médiévale, segmentation, variante, diachronie, hagiographie.

\section{Abstract}

As a historical phenomenon, the punctuation is a priori one subject of diachronic studies. To investigate the variation of same texts' marked pauses among their contemporary and subsequent textual witnesses could bring out the pragmatic diversity as well as the

\footnotetext{
*Artículo recibido el 15/09/2020, aceptado el 30/03/2021.
} 
chronological changes of textual segments marking. Focused on a series of narrative texts, this article has as its objectives not only analysing relevant marks' specific usage and their changing systematisation, but also contributing to the punctuation studies' theoretical development aiming at its more extensive utility, which may be helpful for diachronic lexicologists and textual genealogists, besides syntacticians and orthographists.

Keywords: medieval punctuation, segmentation, variant, diachrony, hagiography.

\section{Introduction : cadre théorique et méthodologique}

Il n'existe jamais deux manuscrits identiques. Si la chasse à la variante peut symboliser la discipline philologie - bien évidemment, de manière très partiale - et si c'est dans cette perspective que Bernard Cerquiglini (1989) intitule son histoire critique de cette discipline Éloge de la variante, pourquoi ne pouvons-nous pas considérer les études sur les signes de ponctuation comme étant dédiées à la pause - valeur intrinsèque visuelle des signes suprasegmentaux ${ }^{1}$ insérés dans un flux graphématique, peu importe s'ils sont appliqués en vue de la réception acoustique ou la réception logico-sémantique du flux ${ }^{2}$ ? Pour une ouvre donnée, tout comme le texte qui n'est jamais constant entre ses témoins distincts, les pauses qui jalonnent et fractionnent le texte varient aussi d'une copie à l'autre, souvent remarquablement en ce qui concerne leurs symboles, leurs emplacements et leurs intensités.

L'étude des signes de ponctuation ne se voit jamais marginaliser dans le lignage linguistico-philologique. Pour trouver sa place théorique dans la linguistique historique, la ponctuation intègre l'orthographe dans le plan épistémologique élaboré par LlamasPombo (2017 : 80). Bien que la notion de la phrase soit problématique en théorie linguistique comme en pratique philologique, les rapports disciplinaires entre l'étude de la ponctuation et delui de la phrase sont a priori indéniables et engendrent de multiples travaux éclairants dans le domaine (par exemple, Marchello-Nizia, 1979 ; Lavrentiev, 2010).

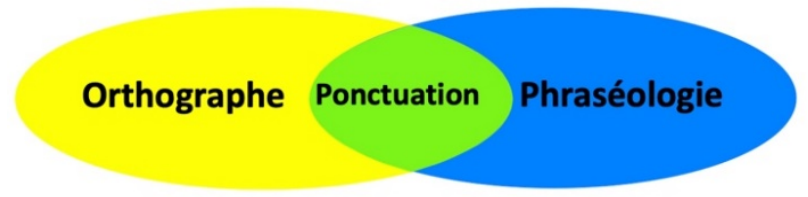

Figure 1. Position de l'étude de la ponctuation.

S'associant à l'orthographe et à la syntaxe de la phrase, la ponctuation ressort en tant que phénomène linguistique historique pour recevoir l'examen de la linguistique variationnelle sous différentes perspectives diasystémiques. Parmi les études

\footnotetext{
${ }^{1}$ Terme de Jacques Anis (1983 : 33), cité par Llamas-Pombo (2017: 45, n. 11).

${ }^{2}$ Pour des exemples concernant ces deux réceptions, voir respectivement la "ponctuation rythmique " et la « ponctuation faible» traitées dans l'article cité de Llamas-Pombo (2017 : 76 et 62).
} 
existantes, la description de l'évolution des emplois du punctus de l'Antiquité à l'époque carolingienne (Llamas-Pombo, 2017 : 62) met en avant l'axe du temps. L'investigation sur la "ponctuation rythmique" (Llamas-Pombo, $2017: 76)$ peut relever l'axe de la forme du texte. Le genre du texte montre son importance dans l'enquête sur la ponctuation « entravée » (Llamas-Pombo, 2017 : 75, 77 ; Mazziotta, 2009). Ce rangement de nos savoirs sur l'histoire de la ponctuation selon diverses perspectives diasystémiques nous amène à apercevoir qu'un autre thème important de la linguistique historique n'est pas encore introduit dans l'étude des signes de ponctuation, à savoir la relation entre la variante et l'évolution.

En diachronie, ce thème ne manque pas d'importance ${ }^{3}$, car il s'agit d'une perspective trans-diasystémique qui tente de décrire les interactions entre les variations diasystémiques non diachroniques et l'évolution chronologique attendue, et de là d'offrir des possibilités, parmi d'autres, pour expliquer la transformation des faits temporairement normatifs. Ayant pour intérêt l'enrichissement de ce thème à la fois transversal et longitudinal, la présente étude se focalisera sur la variation des pauses ponctuées dans un recueil hagiographique inédit, à savoir les Festes nouvelles, entre leurs témoins contemporains et successifs, manuscrits et imprimés. Notre thème privilégie la comparaison des variantes d'un fait linguistique appartenant à un même texte, afin de réduire les variables indépendantes et puis de garantir la comparabilité des données.

\section{Constitution du corpus : la sélection de témoins et le choix de textes}

Largement diffusées à la fin du Moyen Âge et au-delà, les Festes nouvelles nous sont parvenues par quatorze manuscrits datant des $\mathrm{XIV}^{e}$ et $\mathrm{XV}^{e}$ siècles ${ }^{4}$ ainsi qu'une quarantaine d'éditions anciennes qui abondent depuis le dernier quart du $\mathrm{XV}^{\mathrm{e}}$ siècle jusqu'au milieu du siècle suivant (Dunn-Lardeau, 1986 : 293). Si cet ensemble inédit ${ }^{5}$ de Vies de saints composées en moyen français par le traducteur royal Jean Golein (c. 1325-1403) (Bossuat et al., 1964 : 787) à partir de diverses sources hagiographiques $(\mathrm{Na}, 2019 \mathrm{~b}:$ :42-43) nécessite encore des commentaires introductifs, l'ouvrage d'après lequel il est créé et conservé en tant que complément - d'où vient l'épithète nouvelles de ces Festes - est par contre bien connu : c'est la Légende dorée, traduction de Jean de Vignay de la Legenda aurea de Jacques de Voragine ${ }^{6}$. Selon Richard Hamer, Christine Knowles classifie les témoins manuscrits de cette légende en trois groupes (a), (b) et (c),

\footnotetext{
${ }^{3}$ Sur ce thème, voir Combettes (2008), Verjans (2012), Glikman et Verjans (2013) et Na (2019a).

${ }^{4}$ Pour les détails et les références, voir ci-dessous.

${ }^{5}$ Nous en portons un projet d'édition en cours financé par l'Université Sorbonne Nouvelle - Paris 3 et le Centre d'études classiques et médiévales de l'Université de Pékin, en collaboration avec Laura-Maï Dourdy, Lucien Dugaz, Laetitia Sauwala, Michela Spacagno et Pierre Vermander.

${ }^{6}$ Les Festes nouvelles sont créées en tant qu'ajout à la Légende dorée traduite par Jean de Vignay (Bossuat et al., 1964 : 787) et gardent souvent ce statut dans leur reproduction manuscrite (Hamer, $1986: 254$ ) et mécanique (Dunn-Lardeau, $1997: 11$ ).
} 
dont les deux derniers contiennent les Festes nouvelles (Hamer, 1986 : 254). À la liste de Richard Hamer présentant 10 manuscrits du groupe (b) et 3 manuscrits du groupe $(c)^{7}$, il faut ajouter un quatorzième : le manuscrit 452 de la Bibliothèque municipale de Lille, qui présente une version abrégée de nos Festes ${ }^{8}$. Nous nommons ce témoin Ld et nous utiliserons les sigles que donne Richard Hamer pour les manuscrits des groupes (b) et (c) (Hamer, $1986: 254)$.

Étant inédits, ces témoins des Festes nouvelles ne possèdent pas encore de stemma établi, mais les premières études ont montré des liens clairs entre certains d'eux (Hamer, $1986: 254-255)$. En considérant plusieurs facteurs tels que la datation, l'appartenance au groupe ${ }^{9}$, la parenté généalogique et, bien entendu, la densité de ponctuation, nous avons sélectionné les six manuscrits suivants comme documents pour examiner la variation des pauses ponctuées dans la transmission textuelle des Festes:

$\mathrm{Cb}$ : Paris, Bibliothèque nationale de France, français $184, \mathrm{XV}^{\mathrm{e}}$ siècle.

$\mathrm{Db}$ : Paris, Bibliothèque nationale de France, français $242, \mathrm{XV}^{\mathrm{e}}$ siècle.

$\mathrm{Eb}$ : Paris, Bibliothèque nationale de France, français $243, \mathrm{XV}^{\mathrm{e}}$ siècle.

$\mathrm{Gb}$ : Genève, Bibliothèque de Genève, français $57, \mathrm{XIV}^{\mathrm{e}}$ siècle.

Nc: Paris, Bibliothèque de l'Arsenal, 3682-3683, vol. 2, XV siècle.

$\mathrm{Ld}:$ Lille, Bibliothèque municipale, 452, fin XV $\mathrm{XV}^{\mathrm{e}}$ siècle.

Ce sont quatre manuscrits du groupe (b), un du groupe (c), ainsi que le témoin elliptique Ld. Le manuscrit Gb est le seul document daté du XIV siècle (BGE, 2011 : 13), le plus ancien parmi tous les quatorze témoins. Les autres appartiennent tous au $\mathrm{XV}^{\mathrm{e}}$ siècle. Il serait néanmoins inopportun d'exagérer la portée temporelle de ces documents, car nous manquons, pour presque chacun d'eux, de datation précise. Sur le plan généalogique, Eb est probablement copié à partir de $\mathrm{Cb}$ (Hamer, 1986 : 254). Au niveau de la densité de ponctuation, ces six manuscrits choisis sont relativement bien ponctués : les signes et les pauses marquées n'y font pas défaut.

Parmi les témoins imprimés, en privilégiant la diversité diatopique et diachronique, nous avons choisi les quatre éditions suivantes, désormais appelées, pour effectuer notre enquête :

$\alpha \alpha$ : Legende des saints nouveaux, Lyon, Bartholomieu Buyer, 1477.

$\beta \beta$ : s. t., Rouen, Richard Goupil pour Richard Macé et al., 1511.

\footnotetext{
${ }^{7}$ La disparité entre (b) et (c) consiste en les différences de la quantité et de l'ordre des chapitres de la Légende dorée, la différence de la quantité des chapitres des Festes nouvelles ainsi que l'ajout de certains autres textes dans (c) (Hamer, $1986: 254)$.

${ }^{8}$ M. E. Porter et J. H. Baltzell ont signalé ce manuscrit en tant que témoin de la traduction de Jean de Vignay de la Legenda aurea $(1956: 25)$. Nous trouvons que les Festes nouvelles qu'il contient sont en fait abrégées par rapport aux textes conservés dans les autres manuscrits.

${ }^{9}$ La répartition des témoins en groupes de Christine Knowles n'est pas une analyse généalogique, mais un classement selon l'organisation des chapitres du recueil. Voir la note 7.
} 
$\gamma \gamma$ : La legende doree et vie des sainctz et sainctes, Poitiers, Enguilbert de Marnef, 1522.

$\delta \delta:$ La legende doree et vie des sainctz et sainctes, Paris, Jehan Real, 1549.

Les Festes nouvelles regroupent une quarantaine de Vies de saints, dont le nombre exact diffère d'un témoin à l'autre. Nous en tirons la Vie de saint Paulin de Nole, la Vie de saint Fiacre et la Vie de saint Thomas d'Aquin comme échantillons pour réaliser notre sondage sur les signes de ponctuation. Ces trois textes viennent de parties différentes du recueil : Paulin se trouve au milieu ou dans la première moitié (en fonction des témoins qui se structurent différemment), Fiacre à proximité du début du dernier quart, Thomas vers la fin pour la plupart des témoins. Le tableau 1 ci-dessous illustre la présence ou l'absence des textes choisis dans les témoins sélectionnés et démontre l'instabilité de l'organisation du recueil dans la transition de l'époque de production manuelle au temps de l'imprimerie.

\begin{tabular}{|c|c|c|c|c|c|}
\hline \multirow{2}{*}{\multicolumn{2}{|c|}{$\begin{array}{l}\text { Légende } \\
\qquad \begin{array}{l}\text { présence } \\
\text { absence }\end{array}\end{array}$}} & \multirow{3}{*}{$\begin{array}{c}\text { sigle } \\
\mathbf{C b} \\
\end{array}$} & \multicolumn{3}{|c|}{ Vies } \\
\hline & & & \multirow[t]{2}{*}{$\begin{array}{l}\text { Paulin } \\
\text { de Nole }\end{array}$} & \multirow[t]{2}{*}{ Fiare } & \multirow[t]{2}{*}{$\begin{array}{l}\text { Thomas } \\
\text { d'Aquin }\end{array}$} \\
\hline \multirow{10}{*}{ 号 } & \multirow{6}{*}{ 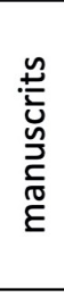 } & & & & \\
\hline & & Db & & & \\
\hline & & Eb & & & \\
\hline & & $\mathbf{G b}$ & & & \\
\hline & & Nc & & & \\
\hline & & Ld & & & \\
\hline & \multirow{4}{*}{ 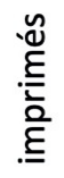 } & $\boldsymbol{\alpha}$ & & & \\
\hline & & $\beta$ & & & \\
\hline & & $\gamma$ & & & \\
\hline & & $\delta$ & & & \\
\hline
\end{tabular}

Tableau 1. Présence / Absence des échantillons dans les témoins choisis.

\section{Objet d'étude : des signes de ponctuation aux signes de segmentation - une redé- finition holiste}

\subsection{Discernement des signes polysémiques ne servant pas à la ponctuation}

Force est de constater que le système de ponctuation évolue considérablement des mains des copistes à celles des typographes. Nous traiterons d'abord les matières manuscrites, qui font preuve d'une homogénéité remarquable dans notre corpus.

La polysémie des signes non alphabétiques n'a rien d'extraordinaire dans les systèmes d'écriture médiévaux, mais cela ne signifie pas que la reconnaissance des signes de ponctuation est toujours un travail facile qui ne demande pas d'effort. Par exemple, dans la figure (2A) ci-dessous, les doubles barres verticales suivant enfant sont en fait deux éléments séparés : la première fait partie de la lettre $t$, la seconde est un signe de ponctuation. Dans (2B), le point au milieu de la ligne suivant la préposition pour abrégée ne ponctue pas le texte, mais rappelle l'existence de l'abréviation. Dans (2C), la 
barre oblique fonctionne comme notre trait d'union moderne, signalant la coupure d'un mot entre deux lignes. Nous affirmons ces faits selon notre observation du système d'écriture de chaque manuscrit concerné :

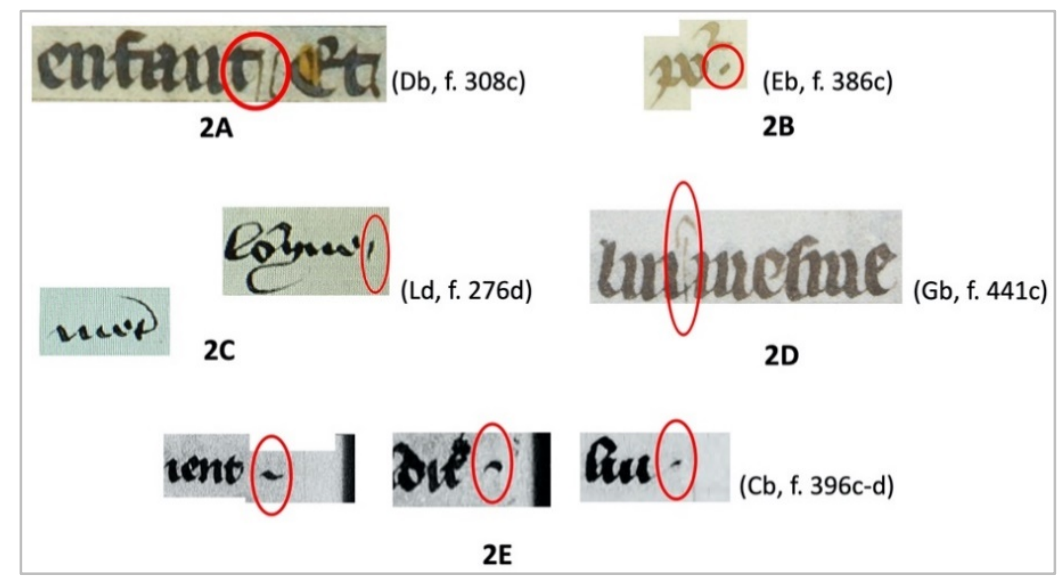

Figure 2. Signes ne servant pas à la ponctuation.

S’il faut préciser que l'identification des marques des (2A-C) appartient aux domaines de la calligraphie et de la paléographie, les marques dans $(2 \mathrm{D})$ et $(2 \mathrm{E})$ refléteront d'autres sphères de l'étude des manuscrits. La barre verticale chapeautée dans (2D) sert à la notation critique, qui déglutine lui et mesme. Dans (2E), le tilde, le petit trait horizontal et le point au milieu de la ligne ne sont pas non plus des signes de ponctuation, bien que les deux premiers se trouvent respectivement à la fin d'une partie textuelle introduite par le pied-de-mouche et à la fin d'une unité phrastique. Dans le système d'écriture de leur document de provenance $\mathrm{Cb}$, ces deux positions syntaxicosémantiques ne réclament pas de signes de ces genres. C'est en fait pour la mise en forme du document que sont ajoutées ces marques. Elles se situent chacune dans un petit espace blanc de la fin d'une ligne (ou, en particulier, de la fin d'une colonne dans le cas du point après lui) qui n'est pas assez grand pour recevoir le premier mot de la ligne suivante. Leur rôle ressemble donc à celui de la décoration fin de ligne de certains manuscrits enluminés.

Pour un recensement exhaustif des signes de ponctuation et l'analyse de leur système, il est avant tout important de discerner les marques qui prennent des formes identiques ou similaires à ces signes mais ne servent pas à leur fin.

\subsection{Hiérarchisation des signes de segmentation}

La ponctuation médiévale " peut comporter, tout comme notre langue actuelle, aussi bien une correspondance qu'une indépendance des signes graphiques par rapport à l'oral » (Llamas-Pombo, 2017 : 63). Pour démontrer le rôle non "rythmique ou respiratoire " de la " ponctuation faible ", les exemples de la virgula marquant la coordination apparue dans des octosyllabes sont convaincants selon des règles phonétiques de la versification syllabique (Llamas-Pombo, 2017: 63). Ce type de signes, bien 
qu'appelés de « liaison » ou d' " enchaînement » dans la terminologie de Llamas-Pombo (2017 : 62), marque de toute façon encore " la sous-division syntagmatique ", comme le signale l'auteur elle-même. Il ne nous semble donc pas imprudent de garder le terme " pause " pour abstraire et décrire la valeur intrinsèque de toutes sortes de signes de ponctuation, à condition de faire distinguer les pauses pour l'œil des pauses pour l'oreille. Dans l'étude citée de Llamas-Pombo (2017 : 62-63), la " pause » est synonyme d'un repos rythmique ou respiratoire de l'oral, alors que le mot distinctio, terme latin du signe de ponctuation, englobe comme connotations la " séparation " des unités syntaxiques «correspondant à une pause de l'oralité », la « liaison » citée plus haut, et le " changement de codes". C'est dans ces deux premiers sens de la distinctio, que nous parlons respectivement des pauses marquées pour l'oreille et pour l'œil, bien qu'en effet, toutes sortes de signes de ponctuation soient avant tout pour l'œil, car il s'agit du processus de la lecture.

Sans changement substantiel $\mathrm{du}$ fond de l'interprétation fonctionnelle des signes de ponctuation auprès de Llamas-Pombo, notre unification terminologique des valeurs des signes de ponctuation en "pause » - rupture visuelle dans un flux graphématique - vise en fait à mettre en avant l'effet de segmentation du texte dans l'usage des signes de ponctuation, étant donné que leur valeur possible de "liaison » correspond encore toujours à quelque sorte de subdivision. Les signes de ponctuation segmentent un texte en repérant des frontières inter-phrastiques et en marquant de diverses relations intra-phrastiques. Pour un grammairien traditionnel, la phrase est « la plus grande unité d'analyse grammaticale "; pour les générativistes, les théories structurelles, les théories dépendancielles ou même la grammaire textuelle, il y a tout de même "une frontière au-delà de laquelle il n'est plus question de dépendance ni de règles combinatoires purement syntaxiques" (Lavrentiev, 2010 : 277). Ce sont des limites des syntacticiens qui ont certainement influencé la réception des signes de ponctuation en linguistique.

Pour les philologues et les codicologues, il est néanmoins essentiel de maintenir un écart avec leurs collègues linguistes afin d'orienter la question vers des sens beaucoup plus concrets et plus réels : la mise en forme et la matérialité. Les signes de ponctuation médiévaux ne sont pas autonomes ou indépendants en tant qu'objet d'observation dans les manuscrits. Leur analyse doit s'intégrer dans le dépouillement du système formel de segmentation du texte mis à l'écrit. Par exemple, les extraits ci-dessous peuvent montrer l'estompage des frontières entre la classe des signes de ponctuation et la classe d'autres signes ou même de certaines mises en forme dans la segmentation d'un flux graphématique :

(1a) / Ains lui va confesser comment il avoit esté evesque Quant le roy le sot il le doubta et tint plus chier que devant [...] (Cb, f. 396c)

(1b) Ains lui va confesser comment il avoit esté evesque Quant le roy le sçeut il le doubta et tint plus chier que devant [...] (Db, f. 309a) 
(1c) / Ains lui va confesser comment il avoit esté evesque // g Quant le roy le sçot / il le doubta et tint plus cher que devant [...] (Eb, f. 387a)

(1d) / Ains lui va confesser comme il avoit esté evesque. Quant le roy le sçeut il le doubta et tint plus chier que devant [...] (Gb, f. 441b)

Tiré de la Vie de saint Paulin, ce morceau possède au milieu une pause interphrastique dans ces quatre manuscrits. Si la pratique de cette coupure dans (1d) correspond à l'attente d'un lecteur moderne - un point au bas de la ligne suivi d'une initiale majuscule de la phrase suivante, la pratique dans (1a) et (1b) - la mise en majuscule de l'initiale en question, sans aucun signe la précédant - pratique récurrente dans des manuscrits médiévaux, montre que la forme majuscule peut jouer à elle seule le rôle du point final. Pour comparer la variation des pauses marquées dans une même tradition textuelle, comment peut-on ignorer les majuscules qui peuvent fonctionner comme des signes de ponctuation ?

La citation (1c) présente, à son tour, un phénomène encore plus intéressant : la variation de l'intensité d'une pause auprès de copistes différents. Celui du manuscrit $\mathrm{Eb}$ applique à la pause en question un pied-de-mouche, signe qui sert à segmenter les épisodes d'une biographie d'un saint dans le système de marquage de ce manuscrit comme dans ceux des autres, alors que les autres copistes ne pensent pas que la narration change ici de thème. On n'est plus en présence d'une question syntaxique, mais plutôt d'une question narratologique. Comment peut-on ignorer cette métamorphose structurelle du texte en étudiant la variation de ses pauses marquées ?

Toutes ces questions nous incitent à adopter une nouvelle attitude à l'égard de la nature des signes de ponctuation. Ils doivent faire parties des signes de segmentation qui englobent encore d'autres marques analytiques du contenu, comme par exemple le pied-de-mouche, et des formes particulières de lettres, par exemple de diverses initiales décorées ou majuscules. Il faut ajouter à cette classe de signes les alinéas ${ }^{10}$, qui sont rares dans les manuscrits mais sont déjà présents dans des imprimés anciens ${ }^{11}$. Tous ces signes forment un système cohérent pour segmenter un texte dans un témoin, système qui est évolutif entre différentes régions et époques, entre différents ateliers et copistesinterprètes. Le noyau du dépouillement de chaque système consiste à éclairer les échelons de la segmentation du texte, ou disons la hiérarchisation des signes de segmentation. La nécessité théorique et la richesse méthodologique de cette perspective peuvent s'inscrire dans la comparaison des variantes de mêmes textes.

\footnotetext{
${ }^{10}$ Nous tenons à remercier Madame Maria Colombo Timelli pour son rappel sur l'alinéa pendant notre discussion au Colloque DIACHRO-IX (Université de Salamanque, mars 2019).

${ }^{11}$ Dans l'imprimé (1477), par exemple, il existe l'alinéa suivi d'un retour à droite pour marquer le début de la rubrique (par exemple, $\alpha, f$. [f viii]b).
} 


\subsection{Attestation de cinq types de signes de ponctuation}

En plus du pied-de-mouche $\langle\mathbf{9}\rangle$, introducteur des plus grandes sections narratologiques d'une $\mathrm{Vie}$, et de l'initiale majuscule (colorée ou non, signalée dans notre transcription respectivement par $<^{*}>$ et $<^{\mathrm{M}}>$ précédant la lettre en question), introducteur des sections de deuxième échelon - correspondant souvent à des unités phrastiques, cinq types de signes de ponctuation sont attestés dans notre corpus manuscrit pour segmenter davantage le texte.

Dans un ordre hiérarchique décroissant, il existe d'abord le point au bas de la ligne $<$. > qui marque dans la plupart des manuscrits, surtout ceux du groupe (b), la fin d'un segment de deuxième échelon. Ensuite, ce sont la barre oblique $</>$ et la barre verticale $<\mid>$ pour séparer les segments de troisième échelon - correspondant souvent à de diverses frontières syntaxiques intra-phrastiques. Pour cette fonction, la barre oblique est le signe le plus fréquemment utilisé dans tous les manuscrits sauf dans Ld - manuscrit elliptique éloigné des autres du point de vue généalogique - où la barre oblique sert à marquer la coupure d'un mot entre deux lignes. En tant que signe nettement différencié de la barre oblique et coexistant avec celle-ci, la barre verticale apparaît seulement dans le manuscrit $\mathrm{Db}$, alors que dans les autres manuscrits, des formes plus ou moins verticales de la barre oblique existent, mais elles sont simplement des variantes graphiques occasionnelles de la barre oblique.

La double barre oblique $</ />$, une sorte de virgula iacens qui « est d'usage courant dans les manuscrits latins et romans comme marque de la division du mot en fin de ligne "(Llamas-Pombo, 2017 : 59), ne remplit que dans Nc cette fonction non pas de ponctuation. Dans les autres codex de notre corpus, ce signe est rarement attesté et joue deux autres rôles distincts. Dans l'échantillon Vie de saint Paulin, il en existe seulement trois occurrences. Les deux premières se trouvent chacune à la fin d'un épisode introduit par le pied-de-mouche, mais tous les autres épisodes de cette Vie ne reçoivent pas ce type de marque finale. Il s'agit en fait encore d'une sorte de signal fin de ligne similaire aux marques de la figure (2E) présentées plus haut dans 2.1. La troisième occurrence, seul cas de $</ />$ en tant que signe de ponctuation dans cette vie, introduit spécifiquement la dernière phrase du texte :

(2) / Puis aprés rendy a Dieu son esperit // Les merites duquel nous soient aydantes in secula seculorum Amen. (Eb, f. 387b)

Il serait inutile d'expliquer l'apparition de cette double barre oblique par la particularité de cette frontière qui est pragmatiquement inter-phrastique, mais peut aussi être intra-phrastique en raison de la fonction de connexion du relatif duquel (fonction issue du relatif de coordination du latin). Nous pouvons trouver dans ce témoin des contre-exemples à foison dans lesquels c'est la barre oblique qui est appliquée à ce genre de pause.

Enfin, pour le cinquième signe de ponctuation attesté dans notre corpus manuscrit, le témoin Nc du groupe (c) témoigne de la survivance de la periodus 
prototypique - le point au haut de la ligne $<^{\cdot}>$. De ce fait, ce codex volumineux du $\mathrm{XV}^{\mathrm{e}}$ siècle conservé à la Bibliothèque de l'Arsenal pourrait se distinguer dans l'histoire des signes de ponctuation, étant donné que la forme dérivée de la periodus, "marque ressemblant au point-virgule moderne ", comme d'autres signes antiques sont déjà " extrêmement rares dans les manuscrits en français à partir du XIII ${ }^{\mathrm{e}}$ siècle " (Lavrentiev, 2010 : 280). Les exemples (3a-b) ci-dessous présentent deux usages typiques de la periodus dans ce manuscrit $\mathrm{Nc}$ :

(3a) Saint fiacre le receut moult benignement ${ }^{\bullet}$ Quant il congnut qu'ilz estoient tous deux d'un pays et conjoinctz ensamble par affinité de sang il lui pria tresaffectueusement qu'il [...] (Nc, f. 290b)

(3b) Tantost il appella un de ses varlets et lui dist ${ }^{\circ}$ vas t'en a cest hostel que tu vois la et fais venir l'ermite secretement parler a moy ${ }^{\circ}$ Le messagier fist ce que l'en lui avoit commandé et [...] (Nc, f. 289a)

Dans la citation (3a), la periodus marque la fin d'une unité phrastique, alors que dans (3b), ce signe ${ }^{12}$ jalonne le début et la fin d'un discours direct. En raison de l'application de la periodus, le point au bas de la ligne $<$. $>$ dans Nc ne possède plus la fonction de marqueur final d'un segment de deuxième échelon comme ceux dans les autres manuscrits. En revanche, il joue un rôle similaire à celui du comma pour marquer une pause intra-phrastique - comme le montre l'exemple (4) ci-dessous - et forme donc avec la periodus un système de ponctuation cohérent et archaïque :

(4) Mon pere et ma mere m'engendrerent en une isle d'Escoce appellee Hybernie. et pour tant que je desire a mener vie solitaire j'ay relenqui mes parens et mon pays et quiers un lieu pour mener vie heremiticque [...] (Nc, f. 289bc)

\subsection{Transcription des données du corpus}

Le tableau 2 ci-dessous récapitule les sept types de signes de segmentation attestés dans notre corpus manuscrit :

\footnotetext{
${ }^{12}$ Il est parfois problématique d'affirmer la position de ce point : en haut ou au milieu de la ligne. Nous décidons enfin de trancher la question en considérant des points vers le milieu de la ligne comme étant des variantes graphiques du periodus, point en haut de la ligne, étant donné que le dépouillement du système de segmentation du manuscrit montre qu'il ne semble pas exister, dans ce document, de niveau de segment comme celui marqué par le signe antique colon, niveau moyen entre le periodus et le comma.
} 


\begin{tabular}{|c|c|}
\hline Pied-de-mouche & $\prod$ (Eb, f. 393d) \\
\hline $\begin{array}{l}\text { Majuscule } \\
\quad \text { (et Maj. colorée) }\end{array}$ & ledde (Db, f. 308c) \\
\hline Point & $11 W$ (Eb, f. 387a) \\
\hline Barre oblique & (Eb, f. 386c) \\
\hline Barre verticale & 12 \\
\hline Double barre oblique & $1 /$ (Eb, f. 387a) \\
\hline Peiodus & (Nc, f. 279d) \\
\hline
\end{tabular}

Tableau 2. Signes de segmentation dans les Festes nouvelles manuscrites

Pour visualiser la variation des pauses marquées entre différents témoins d'un même texte, nous avons choisi au hasard un manuscrit, à savoir Eb, et nous l'avons transcrit comme base de comparaison, puis nous y avons ajouté les signes de segmentation des autres témoins :

3A If ***Quant la femme oÿ dire ces parolles de la propre bouche d'un tel homme comme c'estoit / / elle cuida mieulx qu'il se mocquast d'elle / / que qu'il deist ce

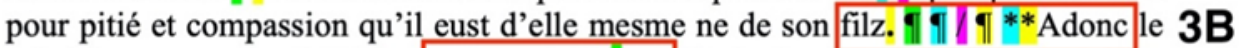

3C saint homme qui estoit bien enlangaigié / et dés le temps de sa jeunesce bien introduis en science. | $* *$ Quant il vit que la femme ne se confioit pas bien en ses parolles / Mtantost lui va dire qu'elle creust fermement ce qu'il lui avoit dit // et que elle ne doubtast / point a mettre en servitude ung evesque pour son filz rachetter// $3 D$ *M*va t'en dist il en aufrique et je yray avec toy $\mid$ I | | **Ainsi ensemble $\mathrm{s}^{\prime} \mathrm{en}$ alerent // ${ }^{* *}$ Et quant ilz furent en aufrique / la femme s'en ala devant le roy / et lui prya qu'elle peust avoir son enfant, \| \ ${ }^{* *}$ Le roy qui estoit moult orguilleux comme ung barbarien $\mid$ ne la daingna oür ne lui respondre $\mid$ | $\mid$ *Adonc de rechief lui dist.. | M*ha sire vez cy ung homme que *je te bailleray en son lieu. | | ** ${ }^{*} \mathrm{Et}$ pourtant aies pitié de moy qui suis une povre femme veufve // et qu'il te plaise de

3E moy rendre mon enfant \|\|$_{\|}^{* *}$ Quant le roy vit le saint homme estre jeune et fort il lui va demander quel mestier il sçavoit faire $\|\mid\|^{*}$ Adonc le saint homme lui Légende: $\square:$ Eb $\square: \mathrm{Db} \quad \square: \mathrm{Cb} \quad \square$ : Gb $\mathrm{m}:$ minuscule les autres signes principaux : voir le tableau 2

Figure 3. Exemple du corpus transcrit.

Comme le montre la figure 3 ci-dessus, nous nous appuyons sur le changement de couleur du fond des signes pour marquer leurs documents de provenance. Cette méthode de marquage visuel destinée à exposer la modalité des variantes peut être 
remplacée par le procédé de balisage en langue TEI dans un futur encodage des données du corpus en vue d'une exploration plus approfondie.

\section{Résultats I : les pauses marquées dans les témoins manuscrits}

\subsection{Conformité et disparité : la liberté du copiste-rubricateur marqueur}

Grâce à notre visualisation polychrome des données, plusieurs caractéristiques de la variation des pauses d'un même texte entre ses témoins différents se manifestent. D'abord, les responsables des documents peuvent être unanimes sur certaines pauses et appliquent le même signe de segmentation. C'est ce que la figure (3A) ci-dessus nous montre. Les copistes-rubricateurs des quatre manuscrits du groupe (b) considèrent tous que la narration tourne une nouvelle page à partir de Quant la femme ö̈ dire... et ils le marquent sans exception par un pied-de-mouche.

Cependant, comme l'expose la figure 3 dans son intégralité, les divergences d'opinions entre les copistes sont beaucoup plus nombreuses que leurs accords. La disparité a lieu non seulement en ce qui concerne l'intensité des pauses - comme nous l'avons montré plus haut avec les exemples (1a-d) et comme le présente la figure (3E) ci-dessus, mais elle peut aussi arriver quand il s'agit de la complexité du marquage et de la densité de pauses. Par exemple, dans (3B), entre filz et Adonc, le responsable du manuscrit $\mathrm{Gb}$ applique un triple marquage : le point, le pied-de-mouche et la coloration de l'initiale, alors que les autres restent moins compliqués. Dans la figure (3C), entre deux attributs juxtaposés, seulement Eb présente une barre oblique. Cela reflète les sensibilités et les pratiques différentes des copistes envers la pause.

Pour signaler une même pause de même niveau, les copistes peuvent aussi se comporter différemment. Selon la figure (3D) plus haut, tous les copistes du groupe (b) signalent le début d'un discours direct : les uns utilisent la majuscule, les autres recourent à la virgula iacens sous forme de barre oblique.

\subsection{Harmonie familiale : la hiérarchisation des signes de segmentation}

Bien que notre corpus témoigne d'une grande liberté des copistes en tant qu'interprètes qui influencent la lecture du texte via les outils de segmentation, nous pouvons encore en tirer des principes communs sur leur pratique. Le tableau 3 ci-dessous montre les systèmes hiérarchiques des signes de segmentation attestés dans nos manuscrits :

\begin{tabular}{||c|c|c||c|c|c||}
\hline \hline \multicolumn{3}{|c||}{ Eb } & \multicolumn{3}{c||}{ Db } \\
\hline $\begin{array}{c}\text { Niveau du } \\
\text { segment }\end{array}$ & $\begin{array}{c}\text { Début } \\
\text { du seg- } \\
\text { ment }\end{array}$ & $\begin{array}{c}\text { Fin du } \\
\text { segment }\end{array}$ & $\begin{array}{c}\text { Niveau du } \\
\text { segment }\end{array}$ & $\begin{array}{c}\text { Début du } \\
\text { segment }\end{array}$ & $\begin{array}{c}\text { Fin du } \\
\text { segment }\end{array}$ \\
\hline grand & $\mathbf{g}$ & & grand & $\mathbf{g}$ & \\
\hline moyen & $\mathrm{M}$ & $\cdot$ (rare) & moyen & $*$ &. (rare) \\
\hline petit & & $/$ & petit & & $/ \quad \mid$ \\
\hline \hline
\end{tabular}




\begin{tabular}{|c|c|c|c|c|c|}
\hline \multicolumn{3}{|c|}{$\mathrm{Cb}$} & \multicolumn{3}{|c|}{$\mathrm{Gb}$} \\
\hline $\begin{array}{l}\text { Niveau du } \\
\text { segment }\end{array}$ & $\begin{array}{c}\text { Début } \\
\text { du seg- } \\
\text { ment }\end{array}$ & $\begin{array}{l}\text { Fin du } \\
\text { segment }\end{array}$ & $\begin{array}{l}\text { Niveau du } \\
\text { segment }\end{array}$ & $\begin{array}{l}\text { Début du } \\
\text { segment }\end{array}$ & $\begin{array}{l}\text { Fin du } \\
\text { segment }\end{array}$ \\
\hline grand & 9 & & grand & 9 & \\
\hline moyen & $\mathrm{M}$ & . (rare) & moyen & * $\quad \mathrm{M}$ & - (fréquent) \\
\hline petit & & 1 & petit & & 1 \\
\hline \multicolumn{3}{|c|}{ Nc } & \multicolumn{3}{|c|}{ Ld } \\
\hline $\begin{array}{l}\text { Niveau du } \\
\text { segment }\end{array}$ & $\begin{array}{l}\text { Début } \\
\text { du seg- } \\
\text { ment }\end{array}$ & $\begin{array}{l}\text { Fin du } \\
\text { segment }\end{array}$ & $\begin{array}{l}\text { Niveau du } \\
\text { segment }\end{array}$ & $\begin{array}{l}\text { Début du } \\
\text { segment }\end{array}$ & $\begin{array}{c}\text { Fin du } \\
\text { segment }\end{array}$ \\
\hline grand & 9 & & grand & 9 & \\
\hline moyen & $\begin{array}{ll}* & \mathrm{M} \\
\end{array}$ & $\cdot$ (parfois) & petit & & . \\
\hline petit & & - (parfois) / (rare) & & & \\
\hline
\end{tabular}

Tableau 3. Systèmes de hiérarchisation des signes de segmentation dans les manuscrits.

Comme nous pouvons le voir sur ce tableau, les manuscrits appartenant au même groupe, classés par Christine Knowles selon l'organisation du contenu des codices, présentent des systèmes similaires pour segmenter le texte. Entre différents groupes, les systèmes se montrent dissemblables et incompatibles. Le manuscrit lillois, témoin elliptique des Festes nouvelles, possède un système encore différent des autres. Les systèmes de segmentation se répartissent en groupes de la même manière que la composition des documents. Ce phénomène est assez intéressant et mérite une attention particulière parmi les généticiens, les philologues et les historiens du livre, car leurs critiques actuelles sur la genèse, la transformation et la transmission d'une œuvre se fondent encore notamment sur le texte - ce qui remplit l'espace d'écrit, et non sur la pause - ce qui épargne l'écrit à l'espace.

\subsection{Variante textuelle et variante de segmentation : quel lien ?}

Coïncidant avec la classification de nos manuscrits selon leurs systèmes de segmentation, la répartition de ces documents en groupes par Christine Knowles est une opération d'après la macrostructure des chapitres du recueil. Si l'on entre dans la comparaison micro-structurelle, c'est-à-dire la variation des détails du texte, que trouverat-on comme rapports entre le regroupement des textes et la discordance des pauses ?

(5a) / Quant le roy l'ot oÿ il le retint moult voulentiers et rendi a la femme vefve son enfant / et elle s'en retourna en son pays moult joyeuse et le saint homme demoura qui moult diligemment mettoit apoint les jardins du roy (Cb, f. 396b)

(5b) Et quant le roy l'eust oÿ / moult volentiers le retint / et rendy a la femme vuefve son enfant / Et quant elle eust son enfant elle s'en retourna en son pays moult joeuse et le saint homme demoura qui moult diligemment mettoit a point les jardins du roy. (Db, f. 308c-d)

(5c) / Quant le roy l'ot oÿ il le reçut moult voulentiers et rendy a la femme veufve son enfant et elle s'en retourna en son pays moult joyeuse / et le saint 
homme demoura qui moult diligenment mettoit a point les jardins du roy (Eb, f. 386c)

(5d) Et quant l'eut oÿ moult volentiers le retint / et rendi a la femme veufve son enfant Et quant elle eut elle se retourna en son pays moult joyeuse et le saint homme demoura qui moult diligenment mettoit a point les jardins $\mathrm{du}$ roy. (Gb, f. 440c-d)

Extraites de la Vie de saint Paulin, les citations (5a-d) proviennent de la fin d'un épisode racontant comment cet évêque de Nole se rend en tant que captif au roi des Vvandres (Eb, f. 386b, etc.) en échange du fils d'une vieille dame veuve. Le roi approuve cet échange et l'enfant retourne avec sa mère dans leur pays. Les variantes micro-structurelles - telles que l'absence contre la présence de la conjonction et au début du morceau, l'ordre des mots le retint/ reçut moult voulentiers contre son inversion moult volentiers le retint, ainsi que la disparition contre la conservation de la subordonnée temporelle Et quant elle eu(s)t (son enfant) - confortent l'opinion de Richard Hamer sur le lien entre $\mathrm{Cb}$ et $\mathrm{Eb}$ mentionnée plus haut dans la section 1. Dans l'autre paire $\mathrm{Db}$ et $\mathrm{Gb}$, le copiste du dernier présente un style relativement plus laconique : il supprime de temps en temps des éléments qui peuvent être entendus selon le contexte, par exemple le sujet le roy dans la première proposition, et le COD son enfant dans la temporelle soulignée en caractères gras.

Dans $(5 a)$ et $(5 c)$, sans cette temporelle, les signes de segmentation suggèrent une juxtaposition d'une phrase complexe et deux propositions indépendantes reliées par deux conjonctions et : (i) quant le roy... il le retint/reçut... et rendily... (ii) ET elle s'en retourna... (iii) ET le saint homme demoura... Dans (5b) et (5d), l'apparition de la temporelle quant elle eu(s)t... qui offre une circonstance précise à la proposition (ii) et pourquoi pas aussi à la proposition (iii), complique davantage la structure de ce morceau qui est présenté comme une seule unité phrastique dans (5a) et (5c). Les copistes de $(5 b)$ et de $(5 d)$ coupent cette unité en deux par la mise en majuscule de l'initiale du mot et qui introduit la proposition (ii) et sa temporelle antéposée. Ce changement de segmentation du texte semble sans aucun doute lié à cette variation textuelle, mais ce rapport ne peut pas être parfaitement prouvé, car l'usage des signes de segmentation n'est presque jamais standardisé dans les manuscrits. Sans cette temporelle, les copistes de $(5 \mathrm{~b})$ et de (5d) peuvent aussi, s'ils veulent, mettre en majuscule l'initiale de la conjonction et en question, conjonction qui possède une sorte de valeur adverbiale comme " puis » dans une narration en moyen français. Avec cette temporelle, les copistes peuvent encore continuer à écrire en minuscule ; le sémantisme ne variera point. C'est seulement le rythme d'enchaînement des évènements qui portera une nuance. 


\subsection{Faible normativité de l'usage des signes : étude de cas du manuscrit Eb}

Divisée en 14 épisodes ${ }^{13}$ par les pieds-de-mouche, la Vie de saint Paulin conservée dans le manuscrit Eb témoigne d'une faible normativité remarquable de l'usage des signes pour marquer les segments du texte de deuxième et de troisième échelons. Cela se traduit par l'application excessive de la barre oblique à diverses pauses de différentes natures et par l'application de divers signes à des pauses de même nature.

Comme le montrent les exemples ci-dessous, à l'intérieur d'une proposition, la barre oblique peut séparer deux attributs juxtaposés (6a) ou deux COD juxtaposés (6b). Ce signe peut être employé entre le prédit et le complément circonstanciel de temps (6c) ou de manière $(6 \mathrm{~d})$ :

(6a) Adonc le saint homme qui estoit bien enlangaigié / et dés le temps de sa jeunesce bien introduis en science / [...] (Eb, f. 386c)

(6b) / Tous les jours saint paulin lui portoit de la poree / et des herbes vertes de son jardin / (Eb, f. 386d)

(6c) [...] comment le royaume des vvandres se doit disposer Car moult honteusement le roy doit mourir / bien brief / Adonc ce seigneur [...] (Eb, f. 386d)

(6d) / Lequel saint homme [...] se fist de son humilité prisonnier pour rachetter les autres prisonniers / en ensuivant l'exemple de nostre seigneur [...] (Eb, f. 387a-b)

Dans les phrases complexes, la barre oblique peut introduire un discours direct (7a) ou une précision explicative (7b). Cette marque peut apparaître entre deux propositions indépendantes coordonnées $(7 \mathrm{c})$, entre deux propositions subordonnées juxtaposées telles que deux complétives ( $7 \mathrm{~d}$ ) ou deux temporelles $(7 \mathrm{e})$, ou entre des subordonnées circonstancielles et la proposition principale (7e) :

(7a) / Adonc de rechief lui dist / ha sire vez cy ung homme que je te bailleray en son lieu / (Eb, f. 386c)

(7b) Le saint homme lui respondy Femme Je n'ay riens que donner maiz fay ainsi / pren moy et dy que je suis ton serviteur affin que tu puisses [...] (Eb, f. 386b-c)

(7c) / Et pourtant aies pitié de moy qui suis une povre femme veufve / et qu'il te plaise de moy rendre mon enfant / (Eb, f. 386c)

(7d) / Quant il vit que la femme ne se confioit pas bien en ses parolles tantost lui va dire qu'elle creust fermement ce qu'il lui avoit dit / et que elle ne

\footnotetext{
${ }^{13}$ Ces épisodes traitent, respectivement, (1) du rachat des prisonniers par Paulin, (2) de la demande d'une veuve, (3) de la réponse de Paulin, (4) de la défiance de la veuve, (5) de la confirmation de Paulin et du rachat du fils de la veuve, (6) du gendre du roi, (7) de l'amitié entre le gendre et Paulin, (8) de l'oracle sur la mort du roi, (9) du choc du roi, (10) de la révélation de l'identité de Paulin, (11) de la réaction du roi, (12) du retour de Paulin et de la libération des captifs, (13) des mérites de Paulin et (14) du trépas de Paulin.
} 
doubtast point a mettre en servitude ung evesque pour son filz rachetter / (Eb, f. 386c) :

(7e) Du temps que / les vvandres gastoient le pays de champaigne et pluseurs autres pays / Et qu'ilz amenoient pluseurs prisonniers de vers les parties auffriquanes / L'omme / de dieu saint paulin evesque / tout ce qu'il povoit avoir de son eveschié il en rachetoit les prisonniers (Eb, f. 386b)

Le manuscrit Eb témoigne aussi d'un emploi de la barre oblique pour introduire l'incise $(7 \mathrm{f})$ :

(7f) Adonc le saint homme qui estoit bien enlangaigié / et dés le temps de sa jeunesce bien introduis en science / Quant il vit que la femme ne se confioit pas bien en ses parolles tantost lui va dire qu'elle creust fermement ce qu'il lui avoit dit / et que elle ne doubtast point a mettre en servitude ung evesque pour son filz rachetter / (Eb, f. 386c)

Dans cette longue citation qui ne possède qu'une seule phrase : Adonc le saint homme ... tantost lui va dire qu'elle ..., la proposition Quant il vit ... parolles, incise entre le sujet saint homme et son prédit lui va dire, est en fait une temporelle donnant une circonstance à l'action va dire. L'adverbe tantost peut être considéré comme une reprise ou un corrélatif de cette temporelle. Du point de vue d'un lecteur moderne, il serait intéressant de remarquer qu'avec la barre oblique, le copiste signale seulement le début mais non la fin de cette incise ${ }^{14}$. En plus, il met en majuscule l'initiale de cette incise. Cette segmentation rejette la première partie du morceau - un sujet, syntagme nominal suivi de sa relative - en dehors de la phrase, ce qui frappera les éditeurs modernes. Si nous abandonnons l'idée de l'incise mais appliquons la perspective de dislocation, c'està-dire qu'il s'agit simplement d'une anaphore de reprise du sujet saint homme par le pronom il dans une phrase complexe normale Quand il ... tantost lui va dire ... ${ }^{15}$, nous serons encore curieux de comprendre la ponctuation en question qui présente le sujet détaché en tant qu'unité phrastique indépendante. Si cette ponctuation n'est pas une erreur, elle reflète bien qu'un copiste médiéval peut s'appuyer sur des principes assez différents des nôtres pour ponctuer un texte. Dans cet exemple, c'est probablement la longueur du flux informationnel, au lieu de la relation sémantico-syntaxique, qui conduit le copiste à segmenter ce morceau en deux unités phrastiques. Le copiste ne considère pas la temporelle comme un élément inséré entre le sujet et son prédit, mais la fusionne avec le prédit et présente, de là, un déroulement unidirectionnel de la narration. Il précise d'abord de qui l'on parle - le sujet, puis raconte ce qui s'est passé sur ce protagoniste - Quant il ... rachetter. La frontière entre ces deux sous-chaînes de l'énoncé

\footnotetext{
${ }^{14}$ Entre parolles et tantost, nous ne voyons pas de signe identifiable selon la numérisation du manuscrit de la BnF. Nous tenons à remercier Mlle Minqi Chu pour son aide dans la vérification de détails du document sur Gallica (qui n'est guère accessible en Chine).

${ }^{15}$ Dans la Vie de saint Paulin, entre la temporelle introduite par quant (toujours antéposée) et la proposition principale, la barre oblique est tantôt présente (9c), tantôt absente (5c).
} 
est signalée comme étant inter-phrastique non seulement dans $\mathrm{Eb}$, mais aussi dans $\mathrm{Db}$ (f. 308c) et Gb (f. 440c). Si l'on interprète ce phénomène comme une erreur commune, cela enrichira nos outils d'analyse dans l'établissement d'un stemma: la comparaison des ponctuations des copistes peut revendiquer une place dans le dépouillement généalogique des témoins, bien que non égale à la comparaison des textes, mais ne devant plus être ignorée. Si ce n'est pas une erreur, le principe derrière cette segmentation partagée entre plusieurs copistes mérite une étude spécifique.

Ce type de segmentation se présente également dans l'extrait (7e) cité plus haut, segmentation qui fractionne une même phrase (du point de vue sémantico-syntaxique) en plusieurs unités phrastiques (selon le système de hiérarchisation des signes de segmentation du manuscrit). Dans (7e), les deux temporelles Du temps que ..., Et qu'ilz..., et la principale L'omme sont présentées en tant que trois unités phrastiques indépendantes. Cette pratique rompt la réservation de la majuscule à la frontière inter-phrastique, que le copiste observe ailleurs, comme par exemple dans la citation (8a) ci-dessous :

(8a) / Le roy qui estoit moult orguilleux comme ung barbarien ne la daingna oÿr ne lui respondre / Adonc de rechief lui dist / ha sire [...] (Eb, f. 386c)

(8b) Adonc le gendre du roy secretement appella le saint homme / et lui demanda qui il estoit. Au quel il respondy qu'il estoit prisonnier du roy [...] (Eb, f. 387a)

Outre la possibilité d'employer la barre oblique pour segmenter deux unités phrastiques introduites par la majuscule, le copiste applique parfois le point au bas de la ligne, comme le présente la citation (8b). Un autre exemple de la faible normativité de l'usage des signes peut être trouvé parmi les discours directs :

(9a) / Adonc le saint homme lui respondy je ne sçay point de mestier [...] (Eb, f. 386c)

(9b) Le saint homme lui respondy Femme Je n'ay riens que donner [...] (Eb, f. 386b)

(9c) Quant le roy le sçot / il le doubta et tint plus chier que devant en lui disant moult humblement / Saige et prudent homme demande telles richesses que tu vouldras avoir pour t'en retourner en ton pays lyement et joyeusement (Eb, f. 387a)

Dans (9a), le copiste n'utilise aucun signe pour introduire le discours direct je ne sçay point ..., alors que dans (7a) plus haut, le copiste emploie une barre oblique. Dans (9b) et (9c), la majuscule est appliquée pour marquer le début du discours direct, mais la barre oblique est tantôt absente (9b), tantôt présente (9c).

Le manque d'une cohérence absolue de la distribution des signes aux diverses pauses caractérise la ponctuation manuscrite médiévale. Le cas du manuscrit Eb en témoigne de manière très manifeste. 


\section{Résultat II : les pauses marquées dans les témoins imprimés}

À la différence des manuscrits, les témoins imprimés datés de 1477, de 1511, de 1522 et de 1549 que nous avons examinés présentent des systèmes homogènes et graduels entre eux pour segmenter les textes :

\begin{tabular}{|c|c|c|c|c|}
\hline $\begin{array}{c}\text { Niveau de } \\
\text { segmentation }\end{array}$ & $\alpha$ & $\mathrm{B}$ & $\Gamma$ & $\delta$ \\
\hline $\mathrm{n}^{\circ} 1$ & $([])^{\mathrm{M}}$ & $([])^{\mathrm{M}}$ & $\mathrm{M}$ & $\mathrm{M}$ \\
\hline $\mathrm{n}^{\circ} 2$ & & $\cdot$ & $\cdot$ & $\cdot$ \\
\hline $\mathrm{n}^{\circ} 3$ & & $:$ ou [] & $:$ & \\
\hline $\mathrm{n}^{\circ} 4$ & & $/$ & $/$ & $/$ \\
\hline
\end{tabular}

Tableau 4. Systèmes de hiérarchisation des signes de segmentation dans les imprimés ${ }^{16}$.

Le tableau 4 ci-dessus illustre les systèmes de hiérarchisation des signes de segmentation conservés dans ces quatre éditions anciennes des Festes nouvelles. Il est remarquable que dans le plus ancien imprimé $\alpha$, aucun signe de ponctuation est appliqué. Il existe seulement la majuscule $\left\langle{ }^{\mathrm{M}}\right\rangle$, précédée d'un espace blanc (symbolisé par [] dans notre transcription) ou non, pour repérer les débuts des unités phrastiques à l'intérieur d'un texte (échantillon examiné : Vie de saint Thomas d'Aquin). Par rapport aux systèmes de trois échelons des manuscrits, nous trouvons dans les imprimés $\beta$ et $\gamma$ un nouveau type de segment marqué par le deux-points $\langle:\rangle$, segment moyen, selon notre hypothèse, plus petit que celui du point mais plus grand que celui de la barre oblique.

(10) Ce saint homme remply du saint esperit vint a la dame a grant joye et luy dist qu'elle avoit conceu ung filz / et elle ne cuidoit pas estre enceinte : adonc le saint homme lui dist. [] Dame esjouys toy [...] ( $\beta$, f. 248b)

Comme le montre la citation (10), dans la narration Ce saint homme ... dist suivie d'un discours direct Dame ..., la plus grande pause doit être la frontière entre cette narration et ce discours, pause marquée par le point suivi d'un espace et d'une initiale majuscule. La pause marquée par le deux-points précédant adonc ... -seconde partie consécutive de la narration - nous semble plus grande que la pause marquée par la barre oblique entre les deux dernières propositions à l'intérieur de la première partie causale de cette narration. Nous présentons ici seulement l'hypothèse d'une fonction intermédiaire du deux-points entre le point et la barre oblique. Dans le texte, des

\footnotetext{
${ }^{16}$ Trois remarques doivent être prises en considération pour lire ce tableau. D'abord, il s'agit d'un module hypothétique se fondant sur l'examen de nos échantillons pour décrire les systèmes de segmentation. Il est probable que d'autres parties des documents présentent des systèmes hétérogènes. Ensuite, nous ne montrons dans ce tableau que des généralités des systèmes. Des exceptions existent; par exemple, l'imprimé possède une occurrence extraordinaire du pied-de-mouche inséré à l'intérieur du texte (f. 234a), phénomène rare selon nos échantillons imprimés. Enfin, les niveaux $n^{\circ} 1$ et $n^{\circ} 2$ se voient de plus en plus fusionner dans les imprimés $\gamma$ et $\delta$. C'est-à-dire que le rôle du point évolue vers un marqueur redondant à la fin de presque chaque unité phrastique commençant par la majuscule.
} 
contre-exemples pourraient exister selon différentes perspectives d'interprétation du fil de la narration.

Dans les imprimés, la normativité de l'usage des signes de segmentation est relativement plus forte que celle des manuscrits. Cela nous permet d'appliquer de simples méthodes quantitatives en vue de résultats valides.

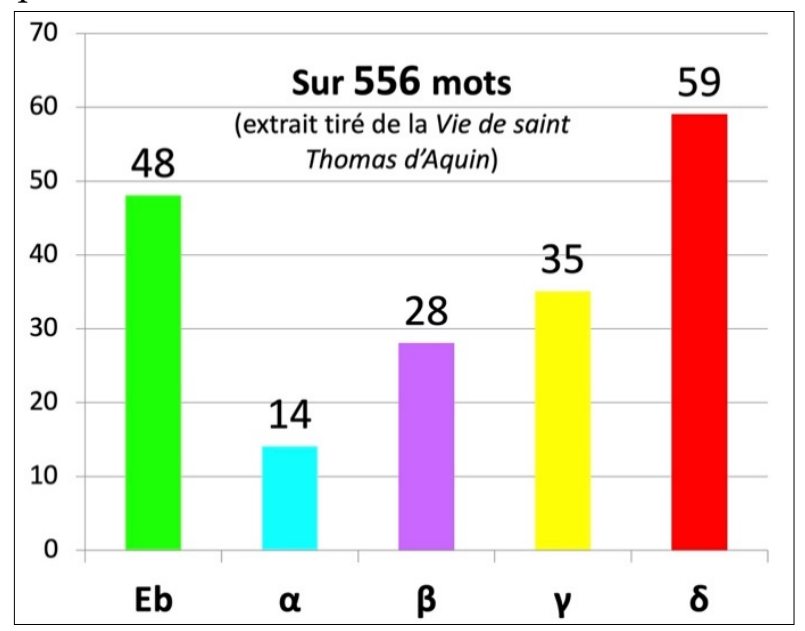

Figure 4. Accroissement continu du nombre des signes de segmentation dans les imprimés.

La figure 4 ci-dessus montre que dans un même espace de texte, à savoir un extrait tiré de la Vie de saint Thomas, plus une édition imprimée est récente, plus elle contient des signes de segmentation. Les trois premiers imprimés possèdent chacun moins de pauses marquées par rapport au manuscrit Eb, alors qu'enfin, le dernier imprimé le surpasse en tant que résultat envisageable suivant cette tendance des typographes à segmenter davantage le texte. Voici une série d'exemples ${ }^{17}$ :

(11a) G Quant l'enfant fu né par son droit nom fu appellé thomas / Le siecle et la vanité $d u$ monde ot / en despit et pour mieulx mener vie sainte et monde il entra en l'ordre des freres prescheurs / et depuis par ses freres charnelz fu ravy et gecté hors / et par deux ans l'enfermerent en une tour fermee . (Eb, f. 402d)

(11b) Quant l'enfant fut né par son droit nom fut thomas appellé le siecle et la vanité $d u$ monde eut en desprisance et pour mieulx mener vie saincte et munde il entra en l'ordre des freres prescheurs et de puis par ses freres charnelz il fut ravi et gecté hors et par deux ans l'enfermerent en une tour fermee $(\alpha, f$. [1 vii]d)

(11c) Quant l'enfant fut né par son droit nom fut thomas appellé [] le siecle et la vanité $d u$ monde eut en desprisant et pour mieulx mener vie saincte et nette il entra en l'ordre des freres prescheurs depuis par ses freres charnelz il fut ravy et getté hors et par deux ans l'enfermerent en une tour fermee . ( $\beta$, f. 248b)

\footnotetext{
${ }^{17}$ En plus des variantes de pause, cette série d'exemples présente aussi des variantes textuelles (lexicales et syntaxiques) intéressantes pour la diachronie. Nous les signalons en italiques.
} 
(11d) Quant l'enfant fut né par son droit nom thomas appellé. Le siecle et la vanité de ce monde eut en despris et pour mieulx mener vie saincte et nette il entra en l'ordre des Freres prescheurs et depuis par ses freres charnelz il fut ravy et getté hors et par deux ans l'enfermerent en une tour fermee. ( $\gamma$, f. 235a) (11e) Quand l'enfant fut ney par son droict nom fut Thomas appellé. Le siecle et la vanité de ce monde eut en despris / et pour mieulx mener vie saincte et necte il entra en l'ordre des freres prescheurs / et depuis par ses freres charnelz il fut ravy et jecté hors / et par deux ans l'enfermerent en une tour bien fermee I ( $\left.\delta, \mathrm{f} .222 \mathrm{a}^{18}\right)$

Ce morceau tiré de la Vie de saint Thomas est considéré comme une seule unité phrastique dans l'imprimé $\alpha$ (1477), mais deux unités dans le manuscrit Eb comme les deux éditions plus récentes $\gamma$ (1522) et $\delta$ (1549). Dans l'édition $\beta$ (1511), le typographe montre une attitude ambiguë en mettant un espace blanc suivi d'une minuscule à la pause en question. Comme nous pouvons l'envisager selon la statistique, le copiste du témoin Eb prend la liberté d'interpréter le texte en recourant à plusieurs types de signes $\langle\mathbf{g}\rangle,\left\langle{ }^{\mathrm{M}}\right\rangle,\langle/\rangle$ et $\langle$. $\rangle$, alors que les responsables des trois premières éditions s'abstiennent d'intervenir trop et ne laissent apparaître que des majuscules et des points finaux. Le dernier typographe du milieu du XVI siècle $(\delta)$ reprend la coutume ancienne d'employer fréquemment la barre oblique. En plus, il applique mieux ce signe que le copiste Eb en l'insérant après en despris (11e) mais non avant (11a). Ensuite, il est remarquable que ce typographe commence à attribuer à la majuscule la valeur de l'initiale de l'anthroponyme, ce qui n'est pas présent dans les autres citations. Enfin, il est encore plus intéressant d'observer les deux occurrences du nom freres dans (11d). Cette citation témoigne d'une grande sensibilité du typographe de $\gamma$ (1522) envers la différence entre le nom commun (freres charnelz) et le nom propre issu du nom commun (Freres prescheurs). Il accorde ici une nouvelle valeur à la majuscule pour signaler cette différence pragmatique.

\section{Une troisième utilité : l'étude de la ponctuation et la lexicologie historique}

En plus d'être liés à l'orthographe et à la syntaxe de la phrase, les signes de ponctuation peuvent contribuer à la lexicologie historique en tant que témoins de l'évolution d'acceptions des mots. Notre corpus possède un exemple significatif :

(12a) [...] il fist livres merveilleux sur toute theologie / logique philosophie / naturelle / moralle / sur les euvangilles / tant que le monde et toute l'eglise de sa sainte science est tout raemply (Eb, f. 403a)

(12b) [...] il fist livres merveilleux sur toute theologie / logique / philosophie naturelle et moralle sur les euvangiles tant que tout le monde est remply de sa science. $(\gamma$, f. 235b)

\footnotetext{
${ }^{18}$ Sur l'imprimé, la pagination est inversée entre « Feuil. CC. xxi. » et « Feuil. CC. xxii. ».
} 
(12c) [...] il feit livres merveilleux sur toute theologie / logicque / philosophie naturelle et moralle / sur les evangiles tant que tout le monde est remply de sa science. $\left(\delta\right.$, f. $\left.222 b^{19}\right)$

Le morceau ci-dessus tiré de la Vie de saint Thomas d'Aquin énumère les thèmes des ouvrages de ce saint docteur. Le contenu de la consécutive introduite par tant que démontre que le syntagme prépositionnel sur les e $(u) v a n g i l(l) e s$ n'est pas un déterminatif des substantifs qui le précèdent, mais est en juxtaposition avec l'autre syntagme prépositionnel plus long sur toute theologie..., sinon le sens de cette consécutive ne sera pas justifié. Dans (12a), ce sont donc six thèmes qui sont mentionnés : theologie, logique, philosophie, naturelle, moralle et euvangilles, mots-clés séparés par la barre oblique, sauf entre logique et philosophie.

Dans (12b) et (12c), le nombre des thèmes devient quatre : theologie, logi(c)que, philosophie naturelle et moralle, et $e(u)$ vangiles. Les signifiés sont structurellement changés, alors qu'au niveau des signifiants, nous voyons simplement l'ajout de la conjonction et et le changement de la ponctuation. Ce n'est pas l'apparition du mot et qui fait entendre cette nouvelle énumération, car cette conjonction ne lie pas forcément deux épithètes du substantif philosophie, si jamais il existe une barre oblique entre philosophie et naturelle dans (12b-c), ces deux mots et le mot suivant moralle seront encore toujours considérés comme étant en juxtaposition. C'est donc la mutation de la ponctuation à elle seule qui change le sens du texte. Dans les deux imprimés $\gamma \gamma$ et $\delta$, l'ajout de la barre oblique entre $\operatorname{logi}(c) q u e$ et philosophie suggère que les typographes de ces documents ont tendance à segmenter les éléments juxtaposés. L'observation de cette pratique n'est par contre pas irréprochable dans (12b), où il manque une barre oblique avant sur les euvangiles. Il est probable que son typographe n'a pas compris la fonction syntaxique de ce syntagme prépositionnel.

Bien que les deux imprimés $\gamma$ et $\delta$, datés respectivement de 1522 et de 1549 , soient postérieurs à $\mathrm{Eb}$, manuscrit du $\mathrm{XV}^{\mathrm{e}}$ siècle, il ne va pas de soi que le texte de (12a) est plus proche de l'authentique que celui de (12b-c). Néanmoins, une variante substantielle entre ces trois témoins peut révéler l'authenticité du manuscrit. La conservation d'un second sujet, toute eglise, dans la consécutive tant que correspond à la répétition de la préposition sur dans la principale, répétition qui répartit les six thèmes en deux groupes, le premier concernant le monde, le second se rapportant à l'eglise. Selon cette cohérence syntaxique et complétude sémantique interne du texte, il est sans doute vrai que la version raccourcie que présentent (12b) et (12c) est postérieure et défectueuse. En plus, leur ponctuation est fautive en ce qui concerne l'énumération des thèmes en question. Ces deux typographes de la première moitié du XVI ${ }^{\mathrm{e}}$ siècle n'ont pas compris les vrais sens des mots naturelle et moralle dans ce texte écrit pas plus tard

${ }^{19}$ Voir la note 18. 
qu'au tournant du XV ${ }^{\mathrm{e}}$ siècle ${ }^{20}$. Ces mots signifient en fait « étude des principes physiques » et "étude des principes éthiques » (DMF) en moyen français du Moyen Âge, alors qu'au XVI ${ }^{\mathrm{e}}$ siècle, ces sens ne sont plus en usage (Huguet). Ces deux substantifs sont de ce fait mal interprétés en tant que deux adjectifs modifiant philosophie par ceux qui ponctuent les éditions $\gamma$ et $\delta$, tels qu'en témoigne la structure syntaxique marquée par les barres obliques qu'ils laissent.

En plus de sa pertinence pour l'orthographe et la syntaxe de la phrase, la ponctuation revendique ici une place en lexicologie historique. À cette troisième utilité, il ne faut pas ignorer non plus son importance possible pour la généalogie philologique, démontrée plus haut dans les sections 3.2 et 3.4.

\section{Conclusion}

Se tenant solidement au milieu des champs de la linguistique historique, l'étude de la ponctuation médiévale peut s'ouvrir davantage en embrassant les matières offertes par ses terrains voisins et consolider, en retour, des édifices de ces domaines contigus. Notre redéfinition holiste des signes de segmentation, qui élargit l'étendue des signes de ponctuation en vue de modéliser plus étroitement les découpages du texte, du plus grand au plus petit, dans un témoin donné, est une orientation requise par la matérialité, c'est-à-dire l'organisation formelle d'un texte dans son support conservé. Cette perspective globaliste, dont la nécessité et l'utilité s'inscrivent profondément dans l'herméneutique philologique, aidera en plus les linguistes à rencontrer de nouveaux phénomènes et à trouver de nouvelles problématiques.

Bien que l'étude venant d'être présentée s'organise autour des signes suprasegmentaux, elle se porte en fait au service des réflexions sur la relation entre la variante et l'évolution, thème d'importance en diachronie. Pour un phénomène évolutif donné, la comparaison des variantes d'un même texte offertes par leurs témoins contemporains et successifs est une méthode fructueuse d'explorer ce thème, comme le montre la variation des pauses marquées dans la transmission des Festes nouvelles.

Dans cette tradition textuelle, le classement des systèmes hiérarchiques des signes de segmentation attestés dans nos échantillons manuscrits correspond au classement de ces documents selon l'organisation macro-structurelle de leur contenu. Au niveau micro-structurel, la variation textuelle peut coïncider avec la variation des pauses marquées, mais rien ne peut en être tiré comme lien nécessaire, puisque la faible normativité est un aspect primordial pour comprendre les pratiques de la segmentation du texte dans nos manuscrits, comme dans la plupart des codices médiévaux.

Lors du passage à l'imprimé, le système de segmentation évolue de façon plus considérable que la mutation du système entre ses variantes au sein de la documentation manuscrite. Selon notre corpus, aux premiers temps de l'imprimerie, les typographes pourraient être assez timides à l'égard de leur droit d'imposer au bloc de texte

${ }^{20}$ L'auteur Jean Golein est mort en 1403 (Bossuat et al., 1964 : 787). 
des repères de différents échelons. Au fur et à mesure que la nouvelle technologie se développe, ses utilisateurs deviennent de plus en plus intrusifs dans la présentation d'un texte, en reprenant des signes hérités de leurs plus proches prédécesseurs, copistes de la fin du Moyen Âge (par exemple, la barre oblique) comme en puisant aux coutumes antiques lointaines certaines marques rarement attestées dans les manuscrits vernaculaires de leur époque précédente (par exemple, le deux-points). L'expansion du marquage des structures sémantico-syntaxiques dans les imprimés pouvait faciliter la promotion de la lecture envers un public de plus en plus large. En répondant à ce besoin venant du bas, un texte devient donc de plus en plus sectionné, morcelé ou même parcellisé dans les moindres détails. La ponctuation et la mise en forme comme la pensée linguistique se conduisent vers une complexité différente de celle du Moyen Âge, à savoir une fragmentation de la Modernité.

\section{RÉFÉRENCES BIBLIOGRAPHIQUES}

ANIS, Jacques (1983) : «Pour une graphématique autonome ». Langue française, 59, 31-44.

BIBLIOTHÈQUE DE GENÈVE (BGE) (2011) : Catalogue des manuscrits français (1-198). $5^{\mathrm{e}}$ édition. Genève, BGE.

Bossuat, Robert; Louis PICHARD \& Guy RaynaUd De LAGE (1964): Dictionnaire des lettres françaises. Le Moyen Âge. Édition entièrement revue et mise à jour sous la direction de Geneviève Hasenohr \& Michel Zink. Paris, Fayard.

CERQUIGLINI, Bernard (1989) : Éloge de la variante : histoire critique de la philologie. Paris, Seuil.

COMBETTES, Bernard (2008) : "Théories du changement et variations linguistiques : la grammaticalisation ». La didactique du français, 137-138, 135-145. URL: https://journals.openedition.org/pratiques/1156

DMF : Dictionnaire du Moyen Français (1330-1500). URL : http://www2.atilf.fr/dmf/

DUNN-LARDEAU, Brenda (1986) : "Étude autour d'une Légende dorée (Lyon, 1476)». Travaux de linguistique et de littérature, 24 : 1, 257-294.

DUNN-LARDEAU, Brenda (1997) : Jacques de Voragine : La Légende dorée. Édition critique, dans la révision de 1476 par Jean Batallier, d'après la traduction de Jean de Vignay (1333-1348) de la Legenda aurea (c. 1261-1266). Paris, Honoré Champion.

GLIKMAN, Julie \& Thomas VerJans (2013) : «Old French Parataxis: Syntactic Variant or Stylistic Variation? ", in Deborah L. Arteaga (éd.), Research on Old French: The State of the Art. Dordrecht, Springer, 243-260.

HAMER, Richard (1986) : "Jean Golein's Festes Nouvelles: A Caxton Source». Medium Aevum, 55, 254-260.

HUGUET, Edmond : Dictionnaire de la langue française du $16^{e}$ siècle. URL : https://classiquesgarnier.com/huguet-dictionnaire-du-xvie-s.html 
LAVRENTIEV, Alexei (2010) : «La "phrase” en français médiéval : une réalité ou une reconstruction artificielle? ", in F. Neveu, V. Muni Toke, J. Durand, T. Klingler, L. Mondada et S. Prévost (éds), Congrès Mondiale de Linguistiques Française - CMLF 2020. Paris, Institut de Linguistique Française, 277-289. URL: http://dx.doi.org/10.1051/cmlf/2010125

LLAMAS-POMBO, Elena (2017) : "Graphie et ponctuation du français médiéval. Système et variation ", in Gabriella Parussa, Maria Colombo Timelli \& Elena Llamas Pombo (éds), Enregistrer la parole et écrire la langue dans la diachronie du français. Tübingen, Narr Francke Attempto, 41-85.

MARCHELLO-NIZIA, Christiane (1979) : «La notion de "phrase" dans la grammaire ». Langue française, 41, 35-48.

MAZZIOTTA, Nicolas (2009) : Ponctuation et syntaxe dans la langue française médiévale. Étude d'un corpus de chartes originales écrites à Liège entre 1236 et 1291. Tübingen, Max Niemeyer.

NA, Yunhao (2019a) : "L'évolution catégorielle des prépositions : enjeu de la morphogenèse des locutions prépositionnelles périphrastiques? Analyse des catégories du mot encontre du XI ${ }^{e}$ au XVI ${ }^{e}$ siècle ", in Daniéla Capin, Julie Glikman, Vanessa Obry \& Thierry Revol (éds), Le français en diachronie. Moyen français - Segmentation des énoncés - Linguistique textuelle. Strasbourg, Éditions de linguistique et de philologie, 315-335.

NA, Yunhao (2019b) : "Les Vies de saint Fiacre du moyen français au français classique : les discordances et la popularisation ". Le moyen français, 84, 35-54.

PORTER, M. E. \& J. H. BAlTZELl (1956) : «The Medieval French Lives of Saint Fiacre». Modern Language Quarterly, 17 : 1, 21-27.

VERJANS, Thomas (2012) : "Le linguiste et la variante : quelle(s) leçon(s) en tirer ? ", in Cécile Le Cornec-Rochelois, Anne Rochebouet \& Anne Salamon (dir.), Le texte médiéval. De la variante à la recréation. Paris, Presses de l'Université Paris-Sorbonne, 89-99. 